\title{
Thermal lensing in recycling interferometric gravitational wave detectors
}

\author{
K.A. Strain ${ }^{1}$, K. Danzmann ${ }^{2}$, J. Mizuno ${ }^{3}$, P.G. Nelson ${ }^{4}$, A. Rüdiger, R. Schilling, W. Winkler \\ Max-Planck Institut für Quantenoptik, Hans-Kopfermann-Strasse 1, D-85748 Garching, Germany
}

Received 17 May 1994; accepted for publication 30 August 1994

Communicated by A. Lagendijk

\begin{abstract}
Thermal lensing limits the performance of advanced interferometric gravitational wave detectors that use high light powers. We evaluate the effects of thermal lensing in such systems and estimate their gravitational wave sensitivity assuming that fused silica optical substrates are employed. Although useful sensitivity can be achieved with established designs, the new technique of resonant sideband extraction is most promising for wideband detectors.
\end{abstract}

\section{Introduction}

Construction of the first generation of laser interferometric gravitational wave observatories is beginning $[1,2]$. In order to improve the range and number of detectable sources, future interferometers should reach integrated strain sensitivities of $\sim 10^{-22}$ over bandwidths of up to $\sim 1 \mathrm{kHz}[1,3]$. This advanced specification requires the use of very high light power, many $\mathrm{kW}$, to reduce the photon shot noise. Absorption of a small fraction of this power in the optical components can lead to serious degradation of the performance of the interferometer [4,5]. In this paper we consider thermal lensing in transmitting optical com-

\footnotetext{
${ }^{1}$ Permanent address: Department of Physics and Astronomy, University of Glasgow, Glasgow G12 8QQ, UK.

${ }^{2}$ Also at Institut für Atom- und Molekülphysik, Universität Hannover, Appelstrasse 2, D-30167 Hannover, Germany.

${ }^{3}$ Now at the Institute of Space and Astronautical Science, 3-1-1 Yoshinodai, Sagamihara-shi, Kanagawa 229, Japan.

${ }^{4}$ Permanent address: TMC, 15 Centenial Drive, Peabody, MA 01960, USA.
}

ponents - the dominant thermal effect in interferometers in which the transmitting optics are made from fused silica (the normal material for the beamsplitter and for the test masses of laser interferometric gravitational wave detectors). The techniques we use can be applied to systems based on other materials and to interferometers dominated by thermal expansion of optical components rather than thermal lensing.

We consider the effect of thermal lensing on a Gaussian laser beam and the implications of the resulting distortion on the stability of an optical cavity containing a thermal lens. We then compare several proposed interferometer configurations to discover which provides the best performance in the presence of thermal lensing. We first summarise the relevant properties of these interferometer designs.

\section{Interferometer configurations}

Fig. 1 shows the essential optics and layout of advanced interferometers. To maximise the signal pro- 


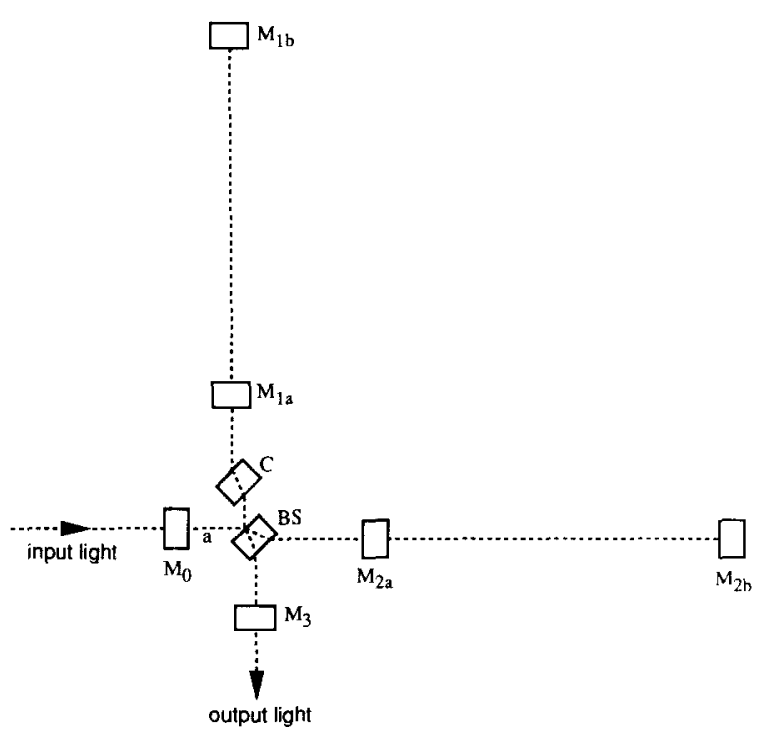

Fig. 1. Optical layout of possible interferometer designs for advanced detectors. The arms may contain either cavities, or delay lines; as shown, the four mirrors $\mathbf{M}_{1 \mathrm{a}}$ to $\mathrm{M}_{2 \mathrm{~b}}$ form two Fabry-Perot cavities. The power recycling mirror $\mathbf{M}_{0}$ allows the light power in the interferometer to be maximised, for a given input power, while the signal recycling/extraction mirror $\mathbf{M}_{3}$ can be used to modify the frequency response of the interferometer and enhance its contrast. The beamsplitter BS and its compensation plate $\mathrm{C}$ are also shown. The light power $P_{0}$, referred to in the text, is that found at point a.

duced by a gravitational wave the interaction time with the light in the interferometer should be half of the period of the wave. Either Fabry-Perot cavities or optical delay lines can be used to extend the light storage times in the arms, which could be several $\mathrm{km}$ long, to match signal periods from $<100 \mu$ s to $>10 \mathrm{~ms}$. Modern low-loss optics allow one to achieve these storage times with little loss of light. With the interferometer operated so that its output remains at a dark fringe most of the light would be reflected back towards the laser and wasted. The addition of a power recycling mirror $\left(\mathrm{M}_{0}\right)$, to form a cavity with the highly reflective interferometer, allows the light power in the arms to be maximised for given input light power and loss [14].

A fundamental limit to the sensitivity of an interferometer is the $\sqrt{n}$ uncertainty associated with the detection of $n$ photons. The shot noise limited sensitivity $\left(h_{\mathrm{min}}\right)$, for an interferometer with power recycling, is (see, e.g., Ref. [3])

$$
\begin{aligned}
h_{\min } & \approx 7 \times 10^{-24}\left(\frac{P_{0}}{10 \mathrm{~kW}}\right)^{-1 / 2}\left(\frac{\Delta f}{100 \mathrm{~Hz}}\right)^{3 / 2} \\
& \times\left(\frac{\lambda}{1064 \mathrm{~nm}}\right)^{1 / 2} .
\end{aligned}
$$

Here $\Delta f$ is the system bandwidth, $P_{0}$ is the light power incident on the beamsplitter from the direction of the laser and $\lambda$ is the wavelength of the light. For best performance $\Delta f$ should be matched to the source bandwidth or measurement bandwidth.

An interferometer in the standard configuration has peak response at low frequency; due to large low frequency noise (seismic noise, etc.) there is unlikely to be any useful low frequency signal and it would be better to tune the interferometer to the most important signal frequencies. This would allow a given strain sensitivity to be achieved with the smallest bandwidth and hence smallest light power. The frequency response of the basic system can be altered using the related techniques of signal recycling [6] or resonant sideband extraction [7].

A frequency domain picture of the operation of the interferometer helps understanding signal recycling and resonant sideband extraction: A suitably polarised gravitational wave phase modulates the "carrier" light stored in the arms of the detector. The modulation index is extremely small and so only the first order sidebands, spaced from the carrier by the gravitational wave frequency, need to be considered. Carrier and sidebands return to the beamsplitter where, with the output maintained at a dark fringe, the carrier light is directed towards the laser and the signal sidebands to the photodetector. A "local-oscillator" would then be used to extract the signal at the original gravitational wave frequency (e.g. using external modulation).

Just as a mirror can be included to "recycle" the carricr light it is possible to add a mirror $\left(\mathrm{M}_{3}\right)$ at the output to "recycle" the signal sidebands. In signal recycling this mirror is chosen to reflect the sidebands back into the system in such a way as to increase the effective overall storage time (they add almost in phase on each return trip of the cavity formed). Signal recycling can be used in interferometers with either delay lines or cavities in the arms to move the peak response from zero frequency to the centre of the detection band. The tuning frequency depends on the phase with which the signal sidebands are reflected 
from the signal recycling mirror (i.e. its position). The bandwidth is determined by the reflectivity of this mirror and the storage time for the light in the arms.

In the interferometer with Fabry-Perot cavities in the arms it is possible to achieve long storage by choosing the correct finesse for the arm cavities, and signal recycling need only to be used to tune the detector to a particular signal frequency. It can be beneficial, however, to use a cavity with a storage time for the carrier rather longer than that required for the signal. This allows the laser power transmitted through optical components to be reduced (for a chosen power in the arms). Resonant sideband extraction was designed to achieve this. The signal-extraction mirror $\left(\mathrm{M}_{3}\right)$ can be arranged to form a coupled cavity system with the arms of the interferometer. Since the carrier light does not reach this mirror it is not affected. The cavity formed by the signal-extraction mirror and the coupling mirrors of the arm cavities can be arranged to be resonant and so to have higher transmission for the signal sidebands than the coupling mirror has for the carrier. The overall coupled cavity thus has a shorter storage time for the signal sidebands than the arm cavities have for the carrier.

An important benefit of either signal technique is improvement of the interferometer contrast [12]. If the two recycling cavities (signal and power) are non-confocal then many higher order modes are nonresonant. Light scattered into these modes, e.g. as a result of deformations of the optics, interferes destructively on successive round-trips in the recycling cavities. To escape from the system the modes thus suppressed have to be transmitted by the recycling mirrors. The loss of power due to the deformations of the optics is therefore significantly reduced.

We introduce the signal recycling factor, $G_{s}$, defined as the ratio of the requested storage time for signals in the interferometer to the storage time for light in the arms. In signal recycling we have $G_{\mathrm{s}}>1$ while in signal extraction $G_{\mathrm{s}}<1$. The effect of $G_{\mathrm{s}}$ on the sensitivity can be appreciated by comparison with the effect of changing the storage-time in the arms directly (by changing the number of beams): While, provided that the total storage time does not exceed the optimum storage time, increasing the number of beams increases storage time and signal proportionally, with signal recycling the signal (amplitude) is enhanced by only $\sqrt{G_{\mathrm{s}}}$. This suggests that we should always try to obtain the greatest possible storage time in the arms to make best use of the power at the beamsplitter. The sensitivity is then obtained by modification of (1),

$$
\begin{aligned}
h_{\min } & \approx 7 \times 10^{-24}\left(\frac{P_{0}}{10 \mathrm{~kW}}\right)^{-1 / 2}\left(\frac{\Delta f}{100 \mathrm{~Hz}}\right)^{3 / 2} \\
& \times\left(\frac{\lambda}{1064 \mathrm{~nm}}\right)^{1 / 2} G_{\mathrm{s}}^{1 / 2} .
\end{aligned}
$$

We use this expression to compare interferometer types after evaluating the power limit imposed by thermal lensing.

\section{Thermal lensing: introduction}

We evaluate the effect of thermal lensing in the transmitting components of the interferometer: the beamsplitter ( $B S$ ) and, if present, the arm-cavity coupling mirrors $\left(M_{1}\right.$ and $\left.M_{2}\right)$. We assume that Gaussian profile laser beams heat the optical substrates through either uniform bulk absorption or uniform absorption in the coatings, and that the optics have an aperture much larger than the beam diameter. We take the result from our earlier paper [8]: For uniform bulk absorption the temperature profile, $T(r)$, in the substrate is

$T(r)=T_{\mathrm{o}}+\frac{p_{\mathrm{a}}}{4 \pi \kappa} \sum_{k=1}^{\infty}\left(2 \frac{r^{2}}{w^{2}}\right)^{k} \frac{(-1)^{k}}{k k !}$.

Here $p_{\mathrm{a}}$ is the relative power absorbed per unit length and $\kappa$ is the thermal conductivity of the substrate, the radial distance from the optical axis is $r$, the beam radius is $w$ and the temperature is offset by a geometrydependent constant $T_{0}$. End effects were neglected but this expression agrees closely with the complete analysis of Hello and Vinet [5]. The refractive index profile is found by multiplying ( 3 ) by the dependence of the refractive index, $n$, on temperature $(\beta=\mathrm{d} n / \mathrm{d} T)$.

It is useful to have a simple measure of the optical path difference $\delta s$ introduced by the thermal lens, measured between the centre of the beam and one Gaussian radius. This can be derived from (3)

$\delta s \approx 1.3 \frac{\beta}{4 \pi \kappa} P_{\mathrm{a}}$,

where $P_{\mathrm{a}}$ is the total absorbed power. If the heating is due to absorption at the coatings of a component 
then this expression must be modified: the factor 1.3 should be replaced by unity [4].

\section{Thermal lensing: mode conversion}

The effect of the thermal lens (or other imperfections of an optical component in a cavity) can be regarded as the scattering of light from the fundamental cavity mode into higher order spatial modes [9]. The radial nature of the problem suggests the use of the Laguerre-Gaussian cavity modes $\left(L_{p}^{l}\right.$ where $p$ is the radial mode number and $l$ the azimuthal mode number, both positive integers). Since the thermal lensing has no azimuthal dependence we consider only those modes with $l=0$. To simplify the form of the expression further we assume that the distortion occurs near the waist of the original mode (in the typical arrangement of an interferometric gravitational wave detector). The modes are then

$L_{p}^{0}=\sqrt{\frac{2}{\pi w^{2}}} \mathbf{L}_{p} \frac{2 r^{2}}{w^{2}} \operatorname{cxp}\left(-\frac{r^{2}}{w^{2}}\right)$,

where $\mathrm{L}_{p}$ are the Laguerre polynomials. For a description of these modes, and their correct normalisation, see Ref. [10].

The thermal lens can be represented by a radial optical phase profile superimposed on the original beam. The amplitude scattered to higher order modes is given by the overlap integral of each of the modes with the unit amplitude, phase-distorted, fundamental mode,

$A_{m}=\int_{0}^{\infty} L_{m}^{0} L_{0}^{0} \exp [\mathrm{i} \phi(r)] 2 \pi r \mathrm{~d} r$,

where $\phi(r)$ is the radially symmetric optical phase distortion introduced by the thermal lens obtained using (3). If the phase shift across the beam is small then the exponential can be expanded $\left(\mathrm{e}^{x} \approx 1+x\right)$. Dropping the overall phase factor $\mathrm{i}$ due to the pure phase distortion, the above expression then simplifies to

$\left|A_{m}\right|=2 \pi \int_{0}^{\infty} L_{m}^{0} L_{0}^{0} \phi(r) r \mathrm{~d} r$.

This expression can be numerically integrated to find the power converted to higher order modes on a single passage through the thermal lens. While the total relative scattered power depends on the magnitude of the thermal lens, the distribution into the higher order modes does not, provided that the small total phase shift condition is satisfied. From the total power scattered $\approx 93 \%$ goes into the first order, $\approx 6 \%$ into the second order and $\approx 0.04 \%$ into the third order. This information is important when designing the recycling cavities to optimise the rejection of the higher order modes produced by thermal lensing.

\section{Thermal lensing in recycling interferometers}

Thermal lensing can influence a recycling interferometer in two ways: distortion of the wavefront can lead to poor contrast (thus reducing the recycling gain) and, for sufficiently strong thermal lensing, the recycling cavity may become unstable.

\subsection{Reduction of contrast}

If the thermal lensing is stronger in one arm of the interferometer than in the other, the resulting mismatch of the interfering wavefronts produces poor interferometer contrast. Signal recycling (or resonant sideband extraction) can reduce the loss of power when, as with thermal lensing, the modes produced by the distortion mechanism are known and can, by careful choice of the geometry of the recycling cavities, be arranged to be non-resonant. Fortunately, thermal lensing scatters little power into very high order modes from where it could not be recovered by signal recycling.

A "mode-cleaner" (non-confocal Fabry-Perot cavity used as a spatial filter [13]) can be used to prevent the light emerging from the interferometer in the higher order spatial modes from reaching the photodetectors and contributing to the detection shot noise. This type of filter can be made very effective if the particular modes which must be filtered are known. Again these can be arranged to be non-resonant and so strongly attenuated by the cavity while the fundamental Gaussian mode is efficiently transmitted. The higher order modes reflected from the mode cleaning cavity back towards the interferometer are discarded (e.g. by the use of a Faraday isolator) so this method does not, however, allow recovery of the power lost from the output of the interferometer. 
A combination of signal recycling and a modecleaner at the output should prevent significant higher order mode power reaching the photodetectors. There remains the question of loss of power in the fundamental mode: If the magnitudes of the thermal lens in the two arms are unequal then the power returning to the beamsplitter in the fundamental mode from each arm will differ. In signal recycling the fundamental mode can be resonant and therefore the loss from the output can be enhanced (as a signal at zero frequency would be enhanced). This is considered separately for each type of interferometer.

\subsection{Recycling cavity instability}

A mirror with a radius of curvature equal to the length of a cavity has a sagitta, over the Gaussian beam diameter in the cavity, of $\sim \lambda / 2 \pi$. Changes in the effective mirror curvature approaching this value are likely to bring the cavity close to instability, a situation that we would like to avoid. As this degree of distortion is approached the beam sizes change producing poor matching of the modes in the various parts of the interferometer and also leading to excessive diffraction losses at mirrors where spot sizes become enlarged.

We assume that, in the absence of any corrective action, the maximum single-pass thermal lensing that can be tolerated is $\delta s \sim \lambda / 4 \pi$. For fused silica ( $\beta=$ $1.2 \times 10^{-5} \mathrm{~K}^{-1}, \kappa=1.38 \mathrm{~W} / \mathrm{K} \mathrm{m}$ ) and with light of wavelength of $\sim 1 \mu \mathrm{m}$ this corresponds to an absorbed power of $\sim 90 \mathrm{~mW}$.

Prefiguring the optics so that they have the correct optical path with thermal lensing may allow higher power, even to the extent that the optics had to be preheated to bring them initially to the correct temperature profile (e.g. with laser light which is strongly absorbed in the glass). An alternative strategy would be correction of the thermal profile of the optics by providing additional heating to "flatten" the temperature profile.

\section{Estimates of the maximum power}

For each design of interferometer we attempt to evaluate the maximum light power in the recycling cavities limited by tolerable thermal lensing.

\subsection{Delay line interferometers}

In delay line interferometers the thermal lensing occurs in the beamsplitter and its compensation plate. The power can be absorbed in the substrate or the coatings, but with state-of-the-art materials the substrate absorption dominates for beamsplitters that are more than a few centimeters thick. (Mirrors are available with absorption losses of about $10^{-6}$ while the best fused silica absorbs about this much per centimeter.) The maximum power is, from (4),

$P_{\max } \approx \frac{4 \pi \kappa \delta s}{1.4 \beta A_{\mathrm{g}} d_{\mathrm{b}}}$,

where $A_{\mathrm{g}}$ is the absorption per unit length in the beamsplitter substrate, $1.1 d_{\mathrm{b}}$ is the light path in the beamsplitter. (For a fused silica beamsplitter, at an angle of incidence of $45^{\circ}$, the light path is about $10 \%$ longer than the thickness of the beamsplitter. The light traverses this path twice, once in each direction.)

A factor of 2 in the tolerable power is obtained by orientation of the beamsplitter so as to minimise the absorption in its substrate (the light comes first to the $50 \%$ coating). This can also be expressed as follows,

$$
\begin{aligned}
P_{\max } & \approx 5 \mathrm{~kW} \frac{\kappa}{1.4 \mathrm{~W} / \mathrm{K} \mathrm{m}} \frac{\delta s}{\lambda / 4 \pi} \frac{\lambda}{1064 \mathrm{~nm}} \\
& \times\left(\frac{1.1 d_{\mathrm{b}}}{0.1 \mathrm{~m}}\right)^{-1}\left(\frac{\beta}{1.2 \times 10^{-5} \mathrm{~K}^{-1}}\right)^{-1} \\
& \times\left(\frac{A_{\mathrm{g}}}{2 \times 10^{-4} \mathrm{~m}^{-1}}\right)^{-1} .
\end{aligned}
$$

The beamsplitter thickness is close to the practical minimum and the value of $A_{\mathrm{g}}$ is the smallest available for large pieces of fused silica at $1064 \mathrm{~nm}$. The minimum absorption at other useful wavelengths, for gravitational wave detectors, is not accurately known. It is possible to obtain relatively small pieces of fused silica, with reduced $\mathrm{OH}^{-}$content and $\sim 10^{-4} / \mathrm{m}$ absorption at $1064 \mathrm{~nm}[11]$.

\subsection{Fabry-Perot interferometers}

With Fabry-Perot cavities in the arms there will be additional thermal lensing in the substrates of the mirrors near the beamsplitter (the "coupling mirrors") The power at the mirror coatings is enhanced by the 
action of the Fabry-Perot arm cavities. This enhancement depends on the finesse $\mathcal{F}$ or equivalently the effective number of beams, $N_{\text {eff }}=2 \mathcal{F} / \pi$. (This holds for cavities in which the transmission of the coupling mirror dominates over mirror losses including transmission of the other mirror.) The ratio of the power in one cavity to the power entering the interferometer at the beamsplitter is $\frac{1}{2} N_{\text {eff }}$. For a Fabry-Perot based interferometer the power limit is, therefore,

$P_{\max } \approx \frac{4 \pi \kappa}{\beta} \frac{\delta s}{1.3 A_{\mathrm{g}} d_{\mathrm{f}}+\frac{1}{2} N_{\mathrm{eff}} A_{\mathrm{c}}}$,

where $A_{\mathrm{c}}$ is the relative absorption per reflection at the coatings of the coupling mirrors and $A_{\mathrm{g}}$ is the relative absorption per unit length in the substrate material. The total path in the substrates of each arm is $d_{\mathrm{f}}=$ $1.1 d_{\mathrm{b}}+d_{\mathrm{m}}$, where $d_{\mathrm{m}}$ is the thickness of a FabryPerot mirror - again, the light traverses this distance twice. The total path is considerably larger than in the delay-line case.

In order to ease the comparison with delay lines the above expression can be split into two cases, neglecting either term in the denominator. If the finesse of the arm cavities is small then

$$
\begin{aligned}
P_{\max } & \approx 1.5 \mathrm{~kW} \frac{\kappa}{1.4 \mathrm{~W} / \mathrm{K} \mathrm{m}} \frac{\delta s}{\lambda / 4 \pi} \frac{\lambda}{1064 \mathrm{~nm}} \\
& \times\left(\frac{\beta}{1.2 \times 10^{-5} \mathrm{~K}^{-1}}\right)^{1}\left(\frac{A_{\mathrm{g}}}{2 \times 10^{-4} \mathrm{~m}^{-1}}\right)^{-1} \\
& \times\left(\frac{d_{\mathrm{f}}}{0.3 \mathrm{~m}}\right)^{-1}
\end{aligned}
$$

Whereas, if the finesse is high

$$
\begin{aligned}
& P_{\max } \approx 230 \mathrm{~W} \frac{\kappa}{1.4 \mathrm{~W} / \mathrm{K} \mathrm{m}} \frac{\delta s}{\lambda / 4 \pi} \frac{\lambda}{1064 \mathrm{~nm}} \\
& \times\left(\frac{\beta}{1.2 \times 10^{-5} \mathrm{~K}^{-1}}\right)^{-1}\left(\frac{A_{\mathrm{c}}}{10^{-6}}\right)^{-1}\left(\frac{N_{\mathrm{eff}}}{10^{3}}\right)^{-1}
\end{aligned}
$$

In the latter case it is the power in the arm cavities that reaches a limiting value. The two effects contribute equally when

$$
N_{\text {eff }} \approx \frac{2.6 A_{\mathrm{g}} d_{\mathrm{f}}}{A_{\mathrm{c}}} .
$$

The value of $N_{\text {eff }}$ chosen in a particular interferometer design depends on the bandwidth required and is discussed below.

\section{Estimates of sensitivity}

We calculate the sensitivity and input light power corresponding to the maximum power allowed by thermal lensing. It is important to check that thermal lensing does not prevent sufficient interferometer contrast being obtained in each design.

\subsection{Delay line interferometers}

The number of beams, $N$, in delay line interferometers is likely to be limited by the size of the mirrors ( at a number smaller than that required to reach the optimum storage time). Mirrors currently available would allow $\sim 8$ beams in an interferometer with $3 \mathrm{~km}$ arm length. For all bandwidths less than $c / 2 N l$, where $c$ is the speed of light in vacuum and $l$ the armlength, the storage time of the arms is sub-optimum. If signal recycling is used to obtain the optimum signal storage time then

$G_{\mathrm{s}}=\frac{c}{2 N l \Delta f}$.

The limiting sensitivity is found by combining (2) and $(8)$,

$$
\begin{aligned}
h_{\min } & \approx 7 \times 10^{-25} \mathrm{~Hz}^{-3 / 2} \mathrm{~W}^{1 / 2} \Delta f^{3 / 2} \\
& \times \sqrt{\frac{1.4 \beta A_{\mathrm{g}} d_{\mathrm{b}}}{4 \pi \kappa \delta s}}\left(\frac{\lambda}{1064 \mathrm{~nm}}\right)^{1 / 2} \sqrt{G_{\mathrm{s}}} .
\end{aligned}
$$

Substituting for $G_{\text {s }}$ we obtain

$$
\begin{aligned}
h_{\min } & \approx 2 \times 10^{-22} \frac{\Delta f}{200 \mathrm{~Hz}}\left(\frac{\kappa}{1.4 \mathrm{~W} / \mathrm{K} \mathrm{m}}\right)^{-1 / 2} \\
& \times\left(\frac{\beta}{1.2 \times 10^{-5} \mathrm{~K}^{-1}}\right)^{1 / 2}\left(\frac{N}{8}\right)^{-1 / 2}\left(\frac{\delta s}{\lambda / 4 \pi}\right)^{-1 / 2} \\
& \times\left(\frac{A_{\mathrm{g}}}{2 \times 10^{-4} \mathrm{~m}^{-1}}\right)^{1 / 2}\left(\frac{1.1 d_{\mathrm{b}}}{0.1 \mathrm{~m}}\right)^{1 / 2}\left(\frac{l}{3 \mathrm{~km}}\right)^{-1 / 2} .
\end{aligned}
$$

The total relative loss in the interferometer is the sum of the reflection losses at the delay line mirrors, the 
losses at the coatings of the beamsplitter, the Rayleigh scattering in the bulk of the beamsplitter and the loss from the output due to imperfect interference. It is assumed that the last loss can be made small compared to the others (this is necessary if the optimum sensitivity is to be obtained). The total relative loss $A_{\text {tot }}$ can then be estimated,

$$
\begin{aligned}
A_{\mathrm{tot}} & \approx 4 \times 10^{-4} \frac{N}{8} \frac{S_{\mathrm{m}}}{50 \mathrm{ppm}}+2 \times 10^{-4} \frac{S_{\mathrm{b}}}{100 \mathrm{ppm}} \\
& +1.3 \times 10^{-5}\left(\frac{\lambda}{1064 \mathrm{~nm}}\right)^{-4} \cdot \frac{1.1 d_{\mathrm{b}}}{0.1 \mathrm{~m}},
\end{aligned}
$$

where $S_{\mathrm{m}}$ is the loss (scatter plus absorption) per reflection from one of the mirrors in the arms, $S_{\mathrm{b}}$ is the loss per reflection or transmission at the beamsplitter and the last term represents the Rayleigh scattering in the substrates of the beamsplitter and compensation plate. So $A_{\text {tot }} \approx 6 \times 10^{-4}$, in our example. The power recycling gain $G_{\mathrm{p}}$ is given by $1 / A_{\mathrm{tot}}$ and the required input power is $P_{\max } / G_{\mathrm{p}}$, so

$P_{\text {in }}=P_{\text {max }} A_{\text {tot }}$

To obtain $5 \mathrm{~kW}$ at the beamsplitter, we therefore require an input light power of $\sim 3 \mathrm{~W}$.

A tuneable broadband response can be obtained using signal recycling with the peak response tuned to the centre of the signal spectrum and bandwidth about equal to the tuning frequency. The power lost from the output of the interferometer in the fundamental mode (due to asymmetry of the interferometer) may, unfortunately, be increased by signal recycling. It can be shown (see, e.g., Ref. [6]) that, with $\Delta f$ equal to the centre-tuning frequency, this increase is $\sim G_{s} / 8$. The effect of asymmetrical thermal lensing can be found by introducing thermal lensing of extent $\Delta s$ in one arm. The fraction $P / P_{0}$ of the power that remains in the fundamental mode in this arm is, from Ref. [4],

$$
\frac{P}{P_{\mathrm{o}}} \sim\left[1-(\pi \Delta s / \lambda)^{2}\right],
$$

for $\Delta s \ll \lambda / \pi$. The relative power loss $A_{\text {th }}$ from the output of the interferometer can be obtained by destructively interfering two beams that differ in amplitude as a result of the thermal lens in one arm. Relative to the input power, in the absence of signal recycling this gives
$A_{\mathrm{th}} \approx \frac{1}{16}\left(\frac{\pi \Delta s}{\lambda}\right)^{4}$

(Remembering that the input power is twice the power in each arm.) Multiplication by $\frac{1}{8} G_{\mathrm{s}}$ accounts for the enhancement due to signal recycling,

$A_{\mathrm{th}} \approx \frac{G_{s}}{128}\left(\frac{\pi \Delta s}{\lambda}\right)^{4}$

This loss should be small compared to the total loss $A_{\text {tot }}$. We take the value of $G_{\mathrm{s}}$ appropriate to the example delay line interferometer parameters, and compare $\Delta s$ to $\lambda / 4 \pi$, the maximum allowed value of $\delta s$. To have $A_{\text {th }}$ small compared to the estimated value of $A_{\text {tot }} \approx 6 \times 10^{-4}$, we require

$\Delta s \ll 0.9 \frac{\lambda}{4 \pi}\left(\frac{G_{s}}{31}\right)^{-1 / 4}\left(\frac{A_{\mathrm{tot}}}{6 \times 10^{-4}}\right)^{1 / 4}$.

A compensation plate is required, if strong thermal lensing is to be tolerated, but its thermal lensing need only be roughly matched to that of the beamsplitter.

\subsection{Fabry-Perot interferometers}

In Fabry-Perot interferometers a tuneable broadband response can be obtained by choosing an arm finesse approximately half of that which would be used to achieve the same bandwidth with no signal recycling, and adding a signal recycling mirror of $\sim 50 \%$ power transmittance. The bandwidth $\Delta f$ of an interferometer, with Fabry-Perot arm cavities of free spectral range $c / 2 l$, but no signal recycling, is

$\Delta f=\frac{c}{4 l \mathcal{F}}$,

the half-width half-maximum linewidth of one of the arm cavities. To produce an interferometer of approximately the same bandwidth, with a signal recycling factor of 2 , the finesse of the arm cavities should be

$\mathcal{F} \sim \frac{c}{8 l \Delta f}$

The sensitivity with thermal lensing is then 


$$
\begin{aligned}
h_{\min } & \approx 7 \times 10^{-25} \mathrm{~Hz}^{-3 / 2} \mathrm{~W}^{1 / 2} \Delta f^{3 / 2} \\
& \times \sqrt{\frac{\beta G_{\mathrm{s}}}{4 \pi \kappa \delta s}\left(1.3 A_{\mathrm{g}} d_{\mathrm{f}}+\frac{c A_{c}}{8 \pi l \Delta f}\right)} \\
& \times\left(\frac{\lambda}{1064 \mathrm{~nm}}\right)^{1 / 2} .
\end{aligned}
$$

The expression for $h_{\min }^{2}$ with $G_{\mathrm{s}}=2$ is

$$
\begin{aligned}
h_{\mathrm{min}}^{2} & \approx 5 \times 10^{-45}\left(\frac{\Delta f}{200 \mathrm{~Hz}}\right)^{2} \frac{\beta}{1.2 \times 10^{-5} \mathrm{~K}^{-1}} \\
& \times\left(\frac{\kappa}{1.4 \mathrm{~W} / \mathrm{K} \mathrm{m}}\right)^{-1}\left(\frac{\delta s}{\lambda / 4 \pi}\right)^{-1} \\
& \times\left(\frac{\Delta f}{200 \mathrm{~Hz}} \frac{A_{\mathrm{g}}}{2 \times 10^{-4} \mathrm{~m}^{-1}} \frac{d_{\mathrm{f}}}{0.3 \mathrm{~m}}+\frac{1}{4} \frac{A_{\mathrm{c}}}{10^{-6}} \frac{l}{3 \mathrm{~km}}\right) .
\end{aligned}
$$

Unless the bandwidth is considerably smaller than 200 $\mathrm{Hz}$ the thermal lensing duc to absorption in the substrates dominates. The sensitivity with the parameters above would be $h \approx 8 \times 10^{-23}$ with a $200 \mathrm{~Hz}$ bandwidth.

With the design parameters suggested in the above example the total loss in the interferometer would probably be dominated by the loss (mainly scattering) at the Fabry-Perot cavity mirrors. The required input power is then given by

$P_{\mathrm{in}} \approx P_{\max } N_{\mathrm{eff}} S_{\mathrm{m}}$

or, for a detector with $\Delta f$ large enough for substrate absorption to dominate,

$$
\begin{aligned}
P_{\text {in }} & \approx 3 \mathrm{~W}\left(\frac{\Delta f}{200 \mathrm{~Hz}}\right)^{-1}\left(\frac{l}{3 \mathrm{~km}}\right)^{-1} \\
& \times \frac{P_{\max }}{1.2 \mathrm{~kW}} \frac{S_{\mathrm{m}}}{50 \mathrm{ppm}} .
\end{aligned}
$$

So thermally limited performance is achieved with modest input light power.

The loss of power from the output, in the fundamental mode, is less of a problem than in the delay line case, since the signal recycling factor $\left(G_{\mathrm{s}}\right)$ is much smaller. Also, imperfect coupling of the signal sidebands from one cavity to another should have little effect on the sensitivity.

We compare the Fabry-Perot hased interferometer with the delay line system: If the delay line system had the optimum number of beams it could have superior performance with wide bandwidth, as there is less substrate absorption. With smaller than optimum mirrors, and therefore reliance on signal recycling, delay line based interferometers do not perform as well as in the presence of thermal lensing. (From (2) we see that, if the power at the beamsplitter is limited by thermal lensing, the best sensitivity is obtained by minimising the signal recycling factor.)

\subsection{Resonant sideband extraction}

The technique of resonant sideband extraction was proposed specifically to reduce the effect of thermal lensing. In order to ensure that the thermal behaviour is dominated by the thermal lensing due to absorption in the cavity mirror coatings rather than in the substrates or the beamsplitter, and so to benefit from this technique we require

$N_{\text {eff }} \gg \frac{2.6 A_{\mathrm{g}} d_{\mathrm{f}}}{A_{\mathrm{c}}}$

The desired bandwidth sets

$G_{\mathrm{s}}=\frac{c}{2 N_{\mathrm{eff}} l \Delta f}$,

obtained by replacing $N$ by $N_{\text {eff }}$ in (14) above. Combining the last two expressions yields the condition

$$
G_{\mathrm{s}} \ll \frac{A_{\mathrm{c}} c}{5.2 l \Delta f A_{\mathrm{g}} d_{\mathrm{f}}} .
$$

Inserting the parameters used above for the FabryPerot design with signal recycling but choosing a bandwidth of $500 \mathrm{~Hz}$, we obtain

$$
\begin{aligned}
G_{\mathrm{s}} & \ll 0.6 \frac{\Delta f}{500 \mathrm{~Hz}} \frac{A_{\mathrm{g}}}{2 \times 10^{-4} \mathrm{~m}^{-1}} \\
& \times \frac{d_{\mathrm{f}}}{0.3 \mathrm{~m}} \frac{l}{3 \mathrm{~km}}\left(\frac{A_{\mathrm{c}}}{10^{-6}}\right)^{-1} .
\end{aligned}
$$

If this condition is satisfied then the maximum allowed power, from (10) and (30), is

$$
P_{\max } \approx \frac{16 \pi \kappa \delta s l G_{\mathrm{s}} \Delta f}{\beta A_{\mathrm{c}} c}
$$

or 


$$
\begin{aligned}
P_{\max } & \approx 1.5 \mathrm{~kW} \frac{\kappa}{1.4 \mathrm{~W} / \mathrm{K} \mathrm{m}} \frac{\delta s}{\lambda / 4 \pi} \frac{\Delta f}{500 \mathrm{~Hz}} \\
& \times \frac{\lambda}{1064 \mathrm{~nm}}\left(\frac{\beta}{1.2 \times 10^{-5} \mathrm{~K}^{-1}}\right)^{-1}\left(\frac{A_{\mathrm{c}}}{10^{-6}}\right)^{-1} \\
& \times \frac{G_{\mathrm{s}}}{0.6} \frac{l}{3 \mathrm{~km}} .
\end{aligned}
$$

Combining this with (2) we obtain

$$
\begin{aligned}
h_{\text {Iniu }} & \approx 1.5 \times 10^{-22} \frac{\Delta f}{500 \mathrm{~Hz}}\left(\frac{\kappa}{1.4 \mathrm{~W} / \mathrm{K} \mathrm{m}}\right)^{-1 / 2} \\
& \times\left(\frac{\beta}{1.2 \times 10^{-5} \mathrm{~K}^{-1}}\right)^{1 / 2}\left(\frac{\delta s}{\lambda / 4 \pi}\right)^{-1 / 2} \\
& \times\left(\frac{A_{\mathrm{c}}}{10^{-6}}\right)^{1 / 2}\left(\frac{l}{3 \mathrm{~km}}\right)^{-1 / 2} .
\end{aligned}
$$

This shows a factor of $\sim 2$ improved sensitivity over the conventional Fabry-Perot design with the same bandwidth.

For the same bandwidth, arm-length, and mirror losses the input power should be approximately the same as for the conventional Fabry-Perot design (only the power at the beamsplitter is smaller). If the arm cavities have unequal reflectivities some fundamental mode will reach the signal-extraction mirror. This light can be resonant in the signal extraction system and be enhanced. It is therefore necessary to match the thermal lensing in the arms (as with signal recycling). It should be possible to dispense with a compensation plate for the beamsplitter. This would reduce the total losses and required input power slightly.

It is possible to achieve better broadband performance with resonant signal extraction than with the other configurations considered.

\section{Conclusion}

If fused silica remains the material for the transmitting components of laser interferometers then thermal lensing could place a significant limit on detector sensitivity. Improving the contrast with dual recycling, mode-cleaners and careful symmetrising of the interferometer, is important but not sufficient, since the distortion due to thermal lensing limits the power in the interferometer in any case. Although good sensitivity can be achieved with moderate bandwidth, useful for many of the expected signals, it is necessary to reduce the effect of thermal lensing to reach sensitivities appropriate to a "second generation" detector. Resonant sideband extraction is advantageous in this situation. It is important to note that the power limit set by thermal lensing is a problem only for advanced interferometers.

\section{Acknowledgement}

We would like to acknowledge the support of this work by the Bundesministerium für Forschung und Technologie (BMFT) and helpful advice and suggestions from the Glasgow gravitational wave group.

\section{References}

[1] R.E. Vogt et al., in: Proc. Sixth Marcel Grossmann Meeting on General Relativity, eds. H. Sato and T. Nakamura (World Scientific, Singapore, 1992) p. 244.

[2] A. Giazotto et al., The VIRGO project report (1989).

[3] J. Hough et al., Proposal for a joint German-British interferometric gravitational wave detector, Report MPQ 147 , Max-Planck-Institut für Quantenoptik, Garching (1989).

14] W. Winkler, K. Danzmann, A. Rüdiger and R. Schilling, Phys. Rev. A 44 (1991) 7022.

[5] P. Hello and J.-Y. Vinet, Phys. Lett. A 178 (1993) 35 I

[6] B.J. Meers, Phys. Lett. A 142 (1989) 465.

[7] J. Mizuno, K.A. Strain, P.G. Nelson, J.M. Chen, R. Schilling, A. Rüdiger, W. Winkler and K. Danzmann, Phys. Lett. A 175 (1993) 273.

[8] W. Winkler, J.M. Chen, J. Mizuno, P.G. Nelson, A. Rüdiger, R. Schilling, K.A. Strain and K. Danzmann, accepted for publication in Opt. Commun.

[9] W. Winkler, R. Schilling, K. Danzmann, J. Mizuno, A. Rüdiger and K.A. Strain, accepted for publication in Appl. Opt.

[10] Bayer-Helms, Appl. Opt. 23 (1984) 1369.

[11] A. Brillet, private communication (1994).

[12] B.J. Meers and K.A. Strain, Phys. Rev. D 43 (1991) 3117.

[13] A. Rüdiger, R. Schilling, L. Schnupp, W. Winkler, H. Billing and K. Maischberger, Opt. Acta 28 (1981) 641

[14] R.W.P. Drever et al., in: Quantum optics, experimental gravitation, and measurement theory, eds. P. Meystre and M.O. Scully (Plenum, New York, 1983) pp. 503-514 